\title{
A one-step multi-disciplinary VHL clinic: patient benefits and feedback
}

SM O’Toole, L McAndrew, WM Drake, U Srirangalingam

Barts Health WHS NHS Trust

\section{Background}

von Hippel-Lindau (VHL) syndrome is a complex multi-organ disorder with significant associated morbidity and mortality.

VHL patients see a number of different specialists and have many clinic appointments each year. These appointments may be at a number of different hospitals. This traditional approach to care is not best suited to VHL as it is inconvenient and time-consuming for patients. In addition, it has the potential to make clinical decision-making more difficult due to the separation of clinicians.

In 2012 St Bartholomew's Hospital in London set up a new one-stop multidisciplinary VHL clinic. Here we report on its first two years of life.

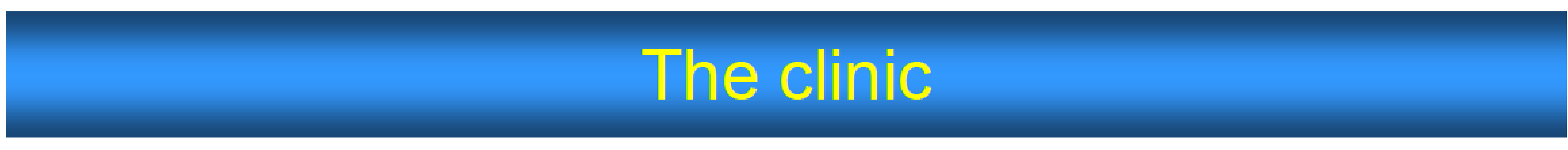

\section{Aims}

- Provide a more comprehensive service and maximise patient benefit from appointments

- Consolidate clinical experience and expertise in the management of VHL

\section{Format}

- Review of all radiology for patients coming to the clinic

- Patients attend in family groups (if desired) and see all clinicians relevant to themselves in one sitting

- Incorporates eye screening

\section{Members}

- Endocrinology (adult and paediatric)

- Genetics

- HPB surgery

- Nephrology

- Neurology

- Neurosurgery

- Oncology

- Ophthalmology

- Psychology

- Radiology

- Urology

- VHL specialist nurse

Methods

Retrospective analysis of paper and electronic records from 2000 until the present day for all VHL patients cared for at St Bartholomew's Hospital, London.

Prospective questionnaire completed by patients attending the new clinic.

\section{Results: demographics}

42 patients are under the care of St Bartholomew's Hospital for VHL; 21 of whom completed the questionnaire.

Mean patient age is 35.4 years (range $10-72$ ) and $58 \%$ are male. The mean distance from the patient's home to the hospital is 30.4 miles (range 2-135).

\section{Results: clinic attendance}

VHL patients attend multiple hospitals frequently for appointments

The mean number of annual attendances to St Bartholomew's Hospital was 4.22 per patient (this did not include scan/phlebotomy visits).

The mean self-reported visit attendance (which also includes appointments at other hospitals) was higher at 7.33. Over three-quarters of patients attended more than one hospital over the course of the year.

VHL patients required fewer clinic appointments following the introduction of the multi-disciplinary clinic

This was significantly less after the introduction of the multi-disciplinary clinic (4.57 $\vee 3.15, p<0.01$ ). All but two patients attended hospital less frequently after the introduction of the clinic.
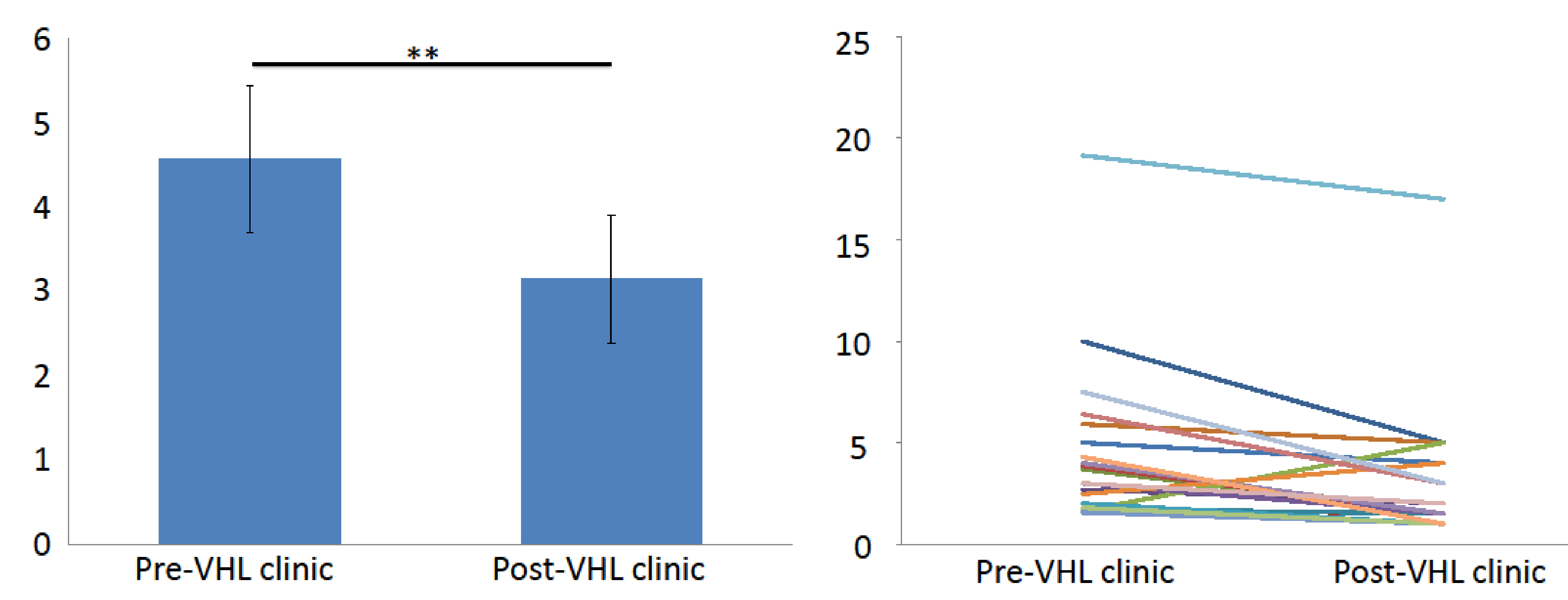

Mean annual hospital attendances before and after the introduction of the one-stop VHL clinic. Displayed for the patient cohort as a whole (left) and on an individual patient basis (right).

\section{Results: patient feedback}

\section{Patient feedback was positive}

All respondents thought a one-stop clinic was a good idea.

95\% preferred to be seen at the same time as their family.

Recurrent themes identified as positive:

- Efficiency

- The team ethos

- Benefit of the specialist nurse as a point of contact and co-ordinato

\section{And provides instruction for service improvement}

Suggestions included:

- Tweaking of organisation of the appointments

- Optional patient support session

\section{Discussion}

An amalgamated one-stop multi-disciplinary VHL clinic is practical to arrange and run and reduces total hospital appointments whilst being well received by patients.

In addition the physical bringing together of all interested parties (both patient and medical) improves communication and allows truly shared decision making. The concentration of clinical material at a single centre also facilitates opportunities for observational and interventional studies.

In today's economic climate, cost effectiveness is an increasingly used and important health metric. This intervention has not yet been evaluated in this regard and might not be 'money-saving' due to the large number of physicians present. 